\title{
A framework design for the mHealth system for self-management promotion
}

\author{
Guifeng Jia ${ }^{\mathrm{a}}$, Pan Yang ${ }^{\mathrm{b}}$, Jie Zhou ${ }^{\mathrm{a}}$, Hengyi Zhang ${ }^{\mathrm{a}}$, Chengyu Lin ${ }^{\mathrm{a}}$, Jin Chen ${ }^{\mathrm{c}}$, Guolong Cai ${ }^{\mathrm{c}}$, \\ Jing Yan $^{\mathrm{c}}$ and Gangmin Ning ${ }^{\mathrm{a}, *}$ \\ ${ }^{a}$ Department of Biomedical Engineering, Key Laboratory of Biomedical Engineering of MOE, \\ Zhejiang University, 310027 Hangzhou, China \\ ${ }^{b}$ Department of Instruments, Hangzhou First People's Hospital, 310006 Hangzhou, China \\ ${ }^{c}$ Department of ICU, Zhejiang Hospital, Lingyin Road 12, 310013 Hangzhou, China
}

\begin{abstract}
Mobile health (mHealth) technology has been proposed to alleviate the lack of sufficient medical resources for personal healthcare. However, usage difficulties and compliance issues relating to this technology restrict the effect of mHealth system-supported self-management. In this study, an mHealth framework is introduced to overcome these drawbacks and improve the outcome of self-management. We implemented a set of ease of use principles in the mHealth design and employed the quantitative Fogg Behavior Model to enhance users' execution ability. The framework was realized in a prototype design for the mHealth system, which consists of medical apparatuses, mobile applications and a health management server. The system is able to monitor the physiological status in an unconstrained manner with simplified operations, while supervising the healthcare plan. The results suggest that the present framework design is accessible for ordinary users and effective in improving users' execution ability in self-management.
\end{abstract}

Keywords: Self-management, mobile health, execution ability, ease of use, persuasive technology

\section{Introduction}

Mobile health (mHealth) is a novel technology that supports self-management to improve health care outcomes [1]. It integrates the technologies of electronics, sensors, mobile computing, communication and medical services [2,3]. This technology is considered an excellent opportunity for health care in developing countries [4,5]. It is widely accepted that mHealth enables: (1) Continuous health monitoring at home, (2) Health self-management, (3) Fewer hospital visits and lower health care cost [6]. Those who suffer from chronic disease or experience inadequate health services will benefit the most from a health care system like this [7, 8].

The mHealth system may provide users self-management; however, some limitations weaken its outcome. Firstly, the integration of multifarious technology in mHealth increases the complexity of functionality and the user interface. Ordinary users, in particular the elderly, may have difficulty operating the mHealth system, which results in obstacles in dealing with self-management [9].

\footnotetext{
* Address for correspondence: Gangmin Ning, Department of Biomedical Engineering, Zhejiang University, Zheda Rd. 38, 310027 Hangzhou, China. Tel.: +86-571-87951091; Fax: +86-571-87951676; E-mail: gmning@zju.edu.cn.
}

0959-2989/15/\$35.00 @ 2015 - IOS Press and the authors. 
Secondly, the outcome of self-management highly depends on the users' ability to execute the suggested healthcare plans. Low executive ability leads to serious compliance issues and depreciates the quality of healthcare. Ensuring the executive ability of users without facing daily consultations is scarcely explored in the field of mobile health [10].

To address these problems, we proposed a framework design for the mHealth system that focuses on promoting the outcome of self-management by enabling user-friendly operation and enhancing users' execution ability. For this purpose, some criteria for the ease of use design are implanted while persuasive technologies are embedded in the system design.

The framework provides an end-to-end solution for health self-management that consists of three units: the medical apparatuses, mobile applications, and health management services. A prototype of the mHealth system, guided by this framework, was developed as an example.

\section{Principals and methods}

\subsection{Ease of use design in the mHealth system}

Ease of use is essential for the mHealth system design. To integrate ease of use principles in the mHealth system and lower complexity in manipulation, four criteria should be considered: accessibility, automation, unconstraint and a user-friendly interface.

Accessibility intends to provide users with direct access to mHealth systems. It is strongly related to universal design. To achieve accessibility, the system must maximize the incorporation of ubiquitous mobile computing and widespread communication technologies. Nowadays, smart phones have come into daily life ubiquitously. Patients and clinicians have a positive attitude toward mobile phone-based health monitoring [11]. Thus, employing smart phones can make the mHealth system easy to access, beside its powerful computation ability. Automation improves the efficiency of user interaction. On account of the diverse capacities of ordinary users to manage devices, the mHealth system must maximize automatic strategies of control and configuration in accordance with the context of use to reduce manual intervention [12]. Unconstraint in this work means that the approach for vital physiological signal acquisition should less boost users' stress or constrained concerns. Non-invasive signal capture is an effective method that simplifies manipulation while bringing users negligible discomfort. In this aspect, significant progress has been made by a number of studies [13, 14]. A user-friendly interface contributes in a natural and intuitive way. It should be easy to read, understand and manage. Using jargon-free language helps users understand interactions clearly, whereas the redundancy design is helpful for user interaction in terms of speed and accuracy [9].

\subsection{Enhancing execution ability design in health self-management}

Persuasive technology can improve users' execution ability and enhance their compliance in daily health care [15]. Persuasive technology is a subdiscipline of Human-Computer Interaction, which is defined as a kind of technology for changing attitudes or behaviors of users through persuasion and social influence [16]. Persuasive technology-supported interventions can be effective and helpful in promoting healthy behavior $[17,18]$.

For embedding persuasive technologies in self-management, the Fogg Behavior Model (FBM) is applied. The FBM is a quantitative model of persuasive technology, which asserts that a target 
behavior requires three key factors: sufficient motivation, ability and effective triggers [19]. Accordingly, to improve the user's execution ability, appropriate triggers and persuasion strategies should be developed to increase motivation, enhance ability, and initiate behavior of users in health management. On the basis of Fogg's work, five strategies were selected to be employed in daily healthcare management. The first is individuation in health care plans. Health status, personal motivation, ability and interests of users are collected and analyzed to make individual health care plans [16]. In this way, the given custom-built healthy tasks would attract users' interests and encourage users to carry them out. The second is self-monitoring. This strategy has two implications. One is that the health care system should be effective in monitoring daily vital physiological signs. In addition, the system is able to evaluate the progress of predetermined tasks during daily health care management. The third is a surveillance strategy. Surveillance means that the system allows a relevant party of users to supervise the implementation of health care plans. This strategy impels users to modify unhealthy behavior in specific manners, for instance, by social networks, which have proved surveillance potential. The fourth is a praise strategy. Providing praise or virtual rewards when daily tasks are achieved will encourage users to steadily improve implementation capacity. The fifth is a reminder strategy. Users who missed tasks will be reminded in convenient ways, such as calling, messages, etc.

\section{Design of mHealth framework}

On the basis of the design principles given in 2.1 and 2.2, the architecture of mHealth framework is supposed and illustrated in Figure 1.

For personal medical apparatuses, we intend to design instruments for vital signals acquisition, including ECG, pulse wave, blood pressure, and body weight. These apparatuses would then deliver the raw data to the mobile applications that are in charge of the medical apparatuses' management, physiological data collection, data processing, features extraction, preliminary analysis, and data transmission. Health management includes users' profile collection and assessment, individualized healthy plan formulation, regular monitoring, and healthcare effectiveness evaluation. To maintain health, the health status of users will be extracted from vital signs and regularly assessed. Health care guidelines help users know how to keep healthy regimens.

In the framework, medical apparatuses and mobile applications interact directly with end-users. Accordingly, ease of use design principles are implemented in them. Meanwhile, persuasive technology is incorporated in health management in the form of a quantitative model to enhance users' execution ability in daily self-management.

\subsection{Personal medical apparatuses design}

The apparatuses consist of physiological sensing, embedded systems for signal processing and wireless communication. Figure 2 illustrates how technical design refers to the ease of use criteria, i.e. unconstraint, automation and accessibility.

For conventional ECG signal acquisition, the electrodes are usually directly placed tightly on the user's body. To achieve an unconstraint design, we presented a creative way that implants the ECG sensors in a chair by installing the electrodes on armrests. On this sensing chair, three electrodes are used: one for the right arm, left arm, and right driving leg. In a similar way, the pulse wave sensor is also installed on the armrest. This non-adherent design has the advantage of comfort and convenience, 


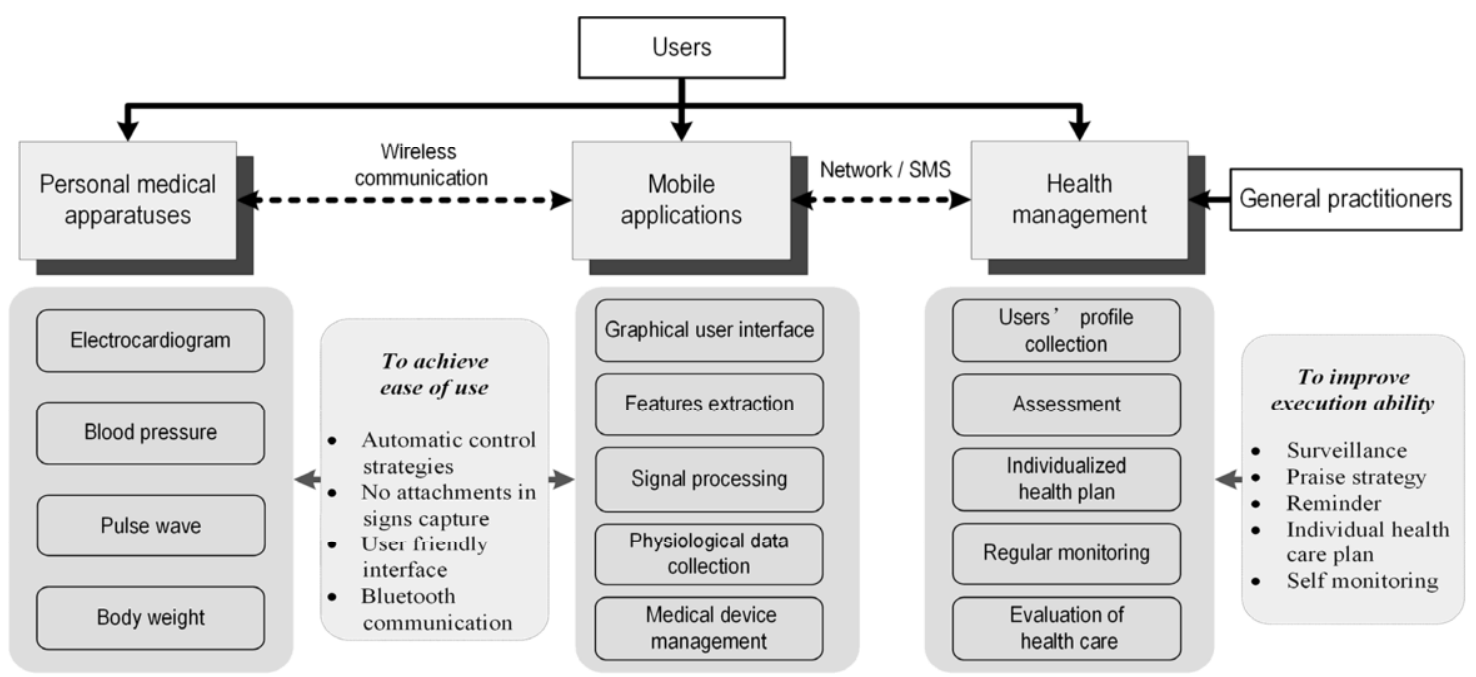

Fig. 1. Architecture of the proposed mobile health care system.

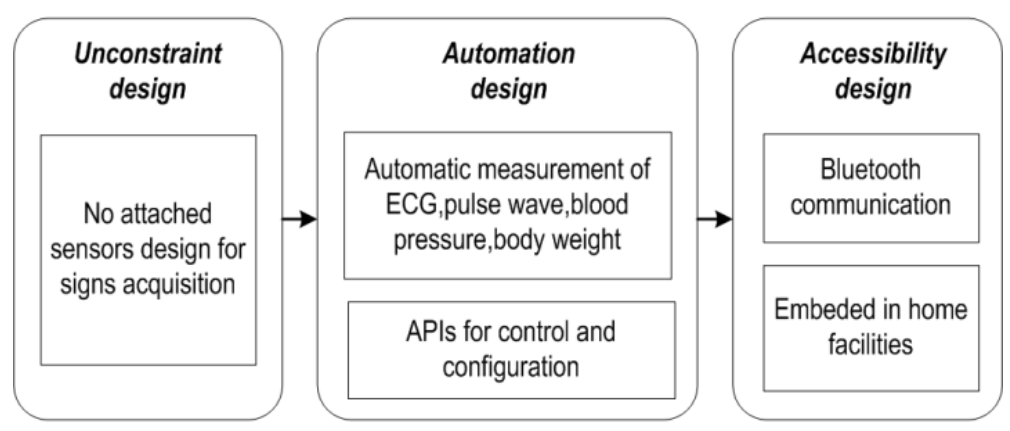

Fig. 2. Ease of use design in personal medical apparatuses.

\begin{tabular}{|c|c|c|c|c|c|}
\hline \multicolumn{2}{|c|}{ Event sensing } & \multicolumn{4}{|c|}{ Graphical user interface } \\
\hline \multicolumn{2}{|c|}{ Automation strategies } & $\begin{array}{l}\text { QRS \& Heartrate } \\
\text { detectlng }\end{array}$ & $\begin{array}{l}\text { Blood pressure } \\
\text { rating }\end{array}$ & BMI rating & \multirow{4}{*}{$\begin{array}{l}\text { Interaction with } \\
\text { remote sever }\end{array}$} \\
\hline \multirow{2}{*}{\multicolumn{2}{|c|}{$\begin{array}{l}\text { Medical apparatus } \\
\text { management }\end{array}$}} & $\begin{array}{l}\text { Detrending \& } \\
\text { denoising }\end{array}$ & \multirow[b]{2}{*}{$\begin{array}{c}\text { Blood pressure } \\
\text { data }\end{array}$} & BMI calculating & \\
\hline & & $\begin{array}{c}\text { ECG/Pulse wave } \\
\text { data }\end{array}$ & & Body weight data & \\
\hline $\begin{array}{l}\text { Apparatus } \\
\text { identify }\end{array}$ & $\begin{array}{l}\text { Control/ } \\
\text { configure }\end{array}$ & \multicolumn{3}{|c|}{ Data transport protocol } & \\
\hline \multicolumn{5}{|c|}{ Bluetooth communication } & Network / SMS \\
\hline
\end{tabular}

Fig. 3. Structure of mobile applications.

which particularly suits elderly people.

Automation requires that a self-acting program instead of manual operation controls the medical apparatuses, to minimize user involvement. Consequently, medical apparatuses are designed to 
support abundance application program interfaces (APIs) for remote procedure call (RPC) services to fulfill the requirement of automatic control and configuration.

\subsection{Mobile applications design}

Mobile applications play a significant role in health care systems involving interaction with users and medical apparatuses, as well as communication with remote servers. The following section describes how ease of use principles are integrated into applications development.

The structure of mobile applications is illustrated in Figure 3. Bluetooth communication is the main channel for data exchange. We built a set of data transport protocols while the apps can identify the medical apparatus type by UID and support automation pairing to the client's Bluetooth device. Such design allows for a flexible plug and play service, which reduces manual intervention. Currently, the available physiological data include ECG, pulse wave, blood pressure and body weight. Accordingly, QRS waves and heart rate are detected. Blood pressure and body mass index (BMI) are graded by a stratification algorithm. The assessment results are presented to users by visualized graphics. With regard to automation criterion, the mobile Apps require a set of adaptive strategies to dominate medical apparatuses based on the context of commands by calling the APIs. We employed the event-driven mechanism in the adaptive strategies, which are implanted in mobile applications. An event is a notable activity indicates triggered by user operation or medical apparatuses. In other words, when the mobile apps identify the coming of a preconcerted event, they will activate the corresponding tasks automatically.

\subsection{Health management integrating with FBM}

The supposed health management is shown in Figure 4. The five persuasive strategies of FBM-namely, investigation, assessment, personalized health plan formulation, self-monitoring, and evaluation - are integrated into the management in a cycle to enhance users' execution ability and adherence.

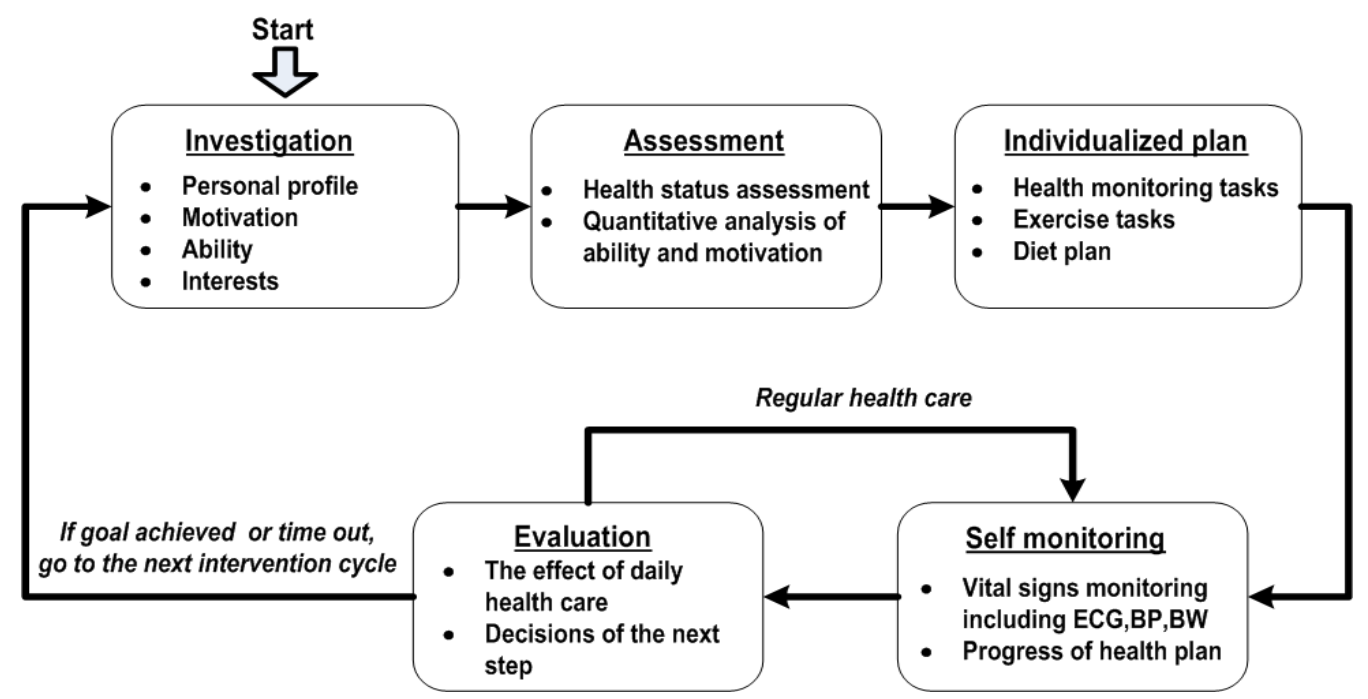

Fig. 4. Health management cycle integrated with persuasive technologies. 


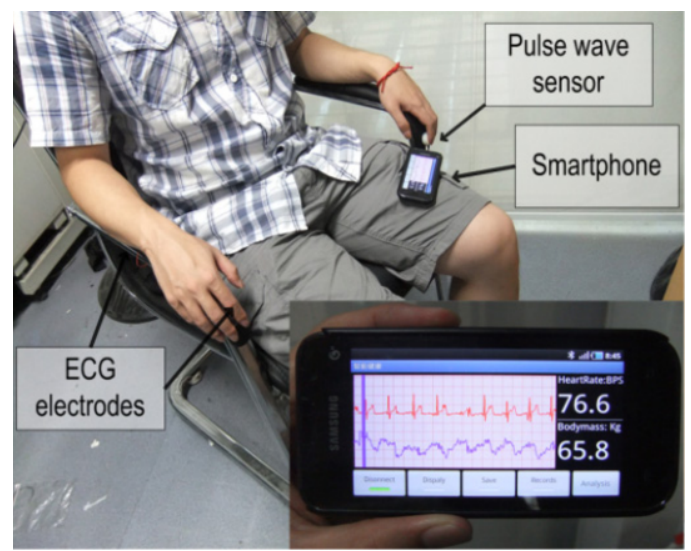

Fig. 5. Sensing chair and mobile app during ECG/pulse acquisition.

The initial stage of the cycle is investigation, which extracts personal information, motivation, ability and interests related to health care. Personal profiles include age, height, body weight and history of disease. Individual motivation, ability and interests are used in building the quantitative FBM. All of the information is collected by questionnaires. The stage of assessment quantitatively analyzes health profiles. According to the collected information, the degrees of user ability and motivation, health status, and lifestyle are assessed. On the basis of the assessment, the third stage generates a personalized healthcare plan, including custom-built exercise tasks, dietary guidance and health suggestions. The stage of self-monitoring is the core of the management. It is designed based on monitoring strategy. Surveillance, reminder and praise strategies are also implemented in this stage. At the evaluation stage, general practitioners evaluate the efficiency of self-management after a fixed period and judge whether the person's health has improved.

\section{Results}

The established framework design was realized in a prototype of a mobile health management system for home use. Medical apparatuses, including a sensing chair, a wireless blood pressure meter and a body scale, were developed. All of them incorporated a client Bluetooth module (2.0) for accessible interaction with mobile apps. In the sensing chair, three custom-built electrodes are embedded in both armrests. While users keep their arms touching the armrests, ECG and pulse signs will be automatically captured. In order to eliminate noise, a filter circuit was designed with a bandpass frequency of $0.7-100 \mathrm{~Hz}$. Also, a digital signal processing algorithm was implemented in the micro-processor.

Three versions of the apps adapted for mobile phones, tablet PCs, and smart TVs were developed on the Android operating system. Figure 5 shows the appearances of the sensing chair, as well as the interface of ECG management in the mobile apps. During the signs capture, ECG, pulse wave and heart rate were represented in real-time. Figure 6 illustrates the interactions of the blood pressure meter and body scale with users and the corresponding interfaces of mobile apps. Body weight management can read the data from the body scale and rate users' BMI. Blood pressure management shows blood pressure and heart rate, while automatically stratificating blood pressure. Color coding is used in management for indicating health status. 
Table 1

Automation strategies in event-driven Apps

\begin{tabular}{ll}
\hline Events & Tasks \\
\hline Apps are activated & Turn on local Bluetooth device, search medical apparatuses \\
Medical apparatuses is found & Identify the type of target medical apparatuses and build a link, \\
& receive data from the apparatuses \\
Depresent the physiological data, save data in local database, send & data to the remote server by SMS or Wi-Fi \\
Apps exit & Disconnect the link and disable local Bluetooth communication \\
\hline
\end{tabular}

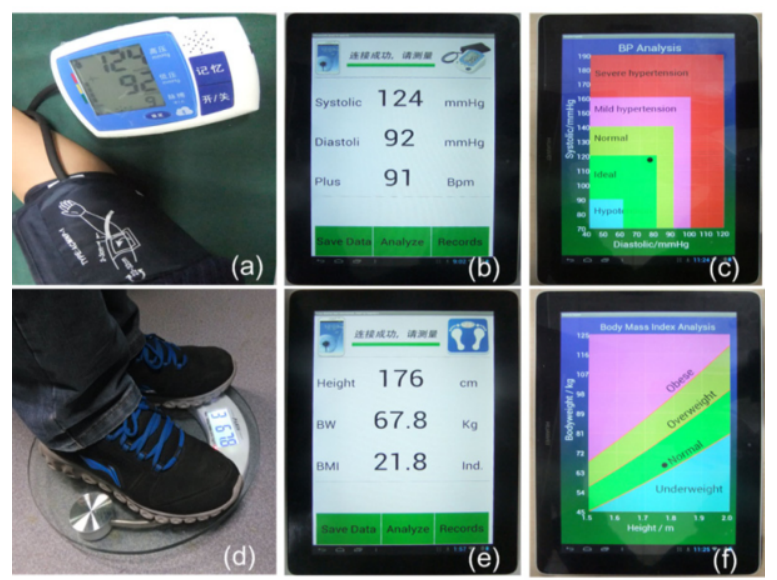

Fig. 6. Outline of health apparatuses and interfaces of mobile apps in tablet PC: (a) Blood pressure measurement with the built blood pressure meter. (b) Interface of blood pressure management. (c) Interface of blood pressure analysis, color-coding represented for health status of blood pressure. (d) Body weight measurement with the built weight scale. (e) Interface of body weight measurement. (f) Interface of body weight analysis.

An event-driven mechanism was applied in the apps, which consisted of an event manager and a service provider. The service provider executes the tasks automatically to respond to the occurred events that were registered in the event manager. In this design, a group of automation strategies were applied; the events and the corresponding tasks are defined in Table 1.

For health management, a web-based server was developed using ASP.NET. MySQL database was employed for data management. The iterative cycle health management model was actualized in the server. The properties of the designed system are summarized in Table 2.

As the core of the health management, the FBM process was realized in the server. Users need to register for access to the health care service. A questionnaire about individual information collects health status, motivation and ability for health improvement, and interests in health-related activities. The information is analyzed, and a personalized health plan is constructed with quantitative goals and tasks. Blood pressure, body weight, ECG and pulse wave during regular monitoring and daily food consumption are collected and stored in the database. The FBM model supervises the users' health plan progress by assessing the difference between the preset goals and the current status. If the daily task is fulfilled, virtual GOLD will be given as rewards; however, when the daily goal is not achieved, reminders or warnings will be present. After a fixed time period, general practitioners evaluate the effect of the current intervention cycle and give feedback information to users; meanwhile, they check the current plan and make necessary modifications to fit the individual status better. The FBM model was tested in a population of 12 volunteer university students. Figure 7 shows an example of a body weight loss plan conducted by the FBM model implemented in a health management website. 
Table 2

The properties of the designed prototype

\begin{tabular}{lll}
\hline Categories & Items & Properties \\
\hline Develop environment & Mobile Apps & Android 4.0 \\
& Web server & ASP.NET \\
\multirow{4}{*}{ Signal Acquisition } & Database & MySQL \\
& Signals & ECG, pulse wave, body weight, blood pressure \\
& Sampling frequency & $250 \mathrm{~Hz}$ \\
Power & Sampling resolution & $12 \mathrm{bit}$ \\
& Power supply & $2 \mathrm{x}$ AA cells \\
Wireless communication & Power consumption & $80 \mathrm{~mW}$ \\
& Protocol & Bluetooth 2.0 \\
& Effective working range & 8 meters (without obstacle) \\
\hline
\end{tabular}

\begin{tabular}{|c|c|c|c|c|}
\hline \multicolumn{2}{|c|}{ Status of completion } & \multicolumn{3}{|c|}{ Rewards } \\
\hline Date & Diet & Water intake & Exercise & Golds \\
\hline 2013-12-20 & +1 & $\mathrm{~N}$ & +3 & 4 \\
\hline 2013.12-19 & $\mathrm{N}$ & +1 & $\mathrm{~N}$ & 1 \\
\hline 2013-12-18 & $\mathrm{N}$ & $\mathrm{N}$ & +3 & 3 \\
\hline 2013-12-17 & +1 & +1 & +3 & 5 \\
\hline 2013-12-16 & +1 & +1 & $\mathrm{~N}$ & 2 \\
\hline 2013-12-15 & $\mathrm{N}$ & $\mathrm{N}$ & $\mathrm{N}$ & 0 \\
\hline 2013-12-14 & $\mathrm{N}$ & +1 & +3 & 4 \\
\hline
\end{tabular}

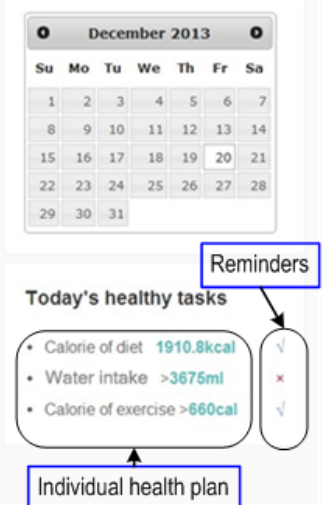

Fig. 7. A web page of the FBM model's supervised individualized health plan.

After the subjects received the supervision of the model for 3 months, $83.3 \%$ of them achieved the preset targets of the health plan. A survey revealed that $66.7 \%$ of the subjects agreed that the FBM model strengthened their motivation for body weight control, while virtual GOLD was considered the most effective reward strategy.

\section{Discussion}

This study presented a framework design for a mobile health system to promote self-management. Despite the endeavors described above, some limitations in this study are obvious. First, in the ECG detector design, the non-adhesive way brings comfort and ease for use, but the sensitivity and dynamic properties have not been assessed yet, compared with clinic investigation. In comparison with commercial devices in clinic, the 12 bits sampling resolution of the ECG signal is relatively low. For the sensing chair design, users' movements during the test may lead to artifacts in the ECG signals, even if the filter was utilized. Also, sitting on a sensing chair may constrain users' mobility during monitoring. Thus, at present, the supposed system is inadequate for diagnosis as in medical institution. Wearable monitoring technology is predicted to be able to compensate for these shortages in future studies [20]. However, it is worth pointing out that the design is not for continuous logging, but for daily self-management, in which a routine test may last only few minutes and vital physiological 
parameters like blood pressure, body weight and heart rhythm are mostly concerned. In this sense, the current work is sufficient for the tasks. Second, medical service is essential for a complete mHealth system. Therefore, the mechanism for connecting users and clinical institutions should be investigated, which is in particular important for emergencies. Besides, for information, exchange electronic medical records (EMR) also should be embedded. These aspects must be taken into account in the improvement of the current work.

\section{Conclusion}

In summary, the remarkable contribution of the work is that it proposed complete solutions to the two main obstacles in self-management, i.e. ease of use and user compliance. A set of design principles for ease of use were introduced to guide the framework design. Meanwhile, the quantitative FBM model was utilized to enhance users' execution ability in self-management. A prototype was successfully developed that covers the fundamental needs of personal health management. The results indicated that the presented framework is valid and helpful in guiding the design of mobile health management systems.

\section{Acknowledgments}

This work is supported by the National Nature Science Foundation of China (Grant 81271662), the Department of Science and Technology of Zhejiang Province (Grant 2011R50018), and the Ministry of Health of China (Grant 201202011).

\section{References}

[1] S. Kumar, W.J. Nilsen, A. Abernethy, et al., Mobile health technology evaluation the mHealth evidence workshop, American Journal of Preventive Medicine 45 (2013), 228-236.

[2] R.S.H. Istepanian, E. Jovanov and Y.T. Zhang, Introduction to the special section on m-Health: Beyond seamless mobility and global wireless health-care connectivity, IEEE Transactions on Information Technology in Biomedicine 8 (2004), 405-414.

[3] H. Demirkan, A smart healthcare systems framework, It Professional 15 (2013), 38-45.

[4] J.G. Kahn, J.S. Yang and J.S. Kahn, 'Mobile' health needs and opportunities in developing countries, Health Affairs 29 (2010), 254-U36.

[5] Vital Wave Consultin, Mhealth for Development: the Opportunity of Mobile Technology for Healthcare in the Developing World, UN Foundation-Vodafone Foundation Partnership, Washington, D.C. and Berkshire, UK, 2009.

[6] H.C. Noel, D.C. Vogel, J.J. Erdos, et al., Home telehealth reduces healthcare costs, Telemedicine Journal and E-Health 10 (2004), 170-183.

[7] M. Chan, E. Campo, D. Esteve, et al., Smart homes - current features and future perspectives, Maturitas 64 (2009), 90-97.

[8] J. Li, H. Zhou, D. Zuo, et al., Ubiquitous health monitoring and real-time cardiac arrhythmias detection: A case study, Bio-medical Materials and Engineering 23 (2013), S1079- S1085.

[9] R.R. Gudur, B. Alethea, P. Vesna and M. Douglas, Redundancy in interface design and its impact on intuitive use of a product in older users, In IASDR 2009 Rigor and Relevance in Design, IASDR 2009, Coex, Seoul, 209.

[10] L.H. Iwaya, M.A.L. Gomes, M.A. Simplicio, et al., Mobile health in emerging countries: A survey of research initiatives in Brazil, International Journal of Medical Informatics 82 (2013), 283-298.

[11] E. Seto, K. J. Leonard, C. Masino, et al., Attitudes of heart failure patients and health care providers towards mobile phone-based remote monitoring, Journal of Medical Internet Research 12 (2010), 56-65. 
[12] A. Lorenz and R. Oppermann, Mobile health monitoring for the elderly: Designing for diversity, Pervasive and Mobile Computing 5 (2009), 478-495.

[13] K.-I. Yamakoshi, Unconstrained physiological monitoring in daily living for health care, Frontiers of Medical and Biological Engineering 10 (2000), 239-259.

[14] D. Qiao, T. He, B. Hu, et al., Non-contact physiological signal detection using continuous wave Doppler radar, Bio-medical Materials and Engineering 23 (2013), S1045-52.

[15] R. de Oliveira, M. Cherubini, N. Oliver, et al., MoviPill: Improving Medication Compliance for Elders Using a Mobile Persuasive Social Game, Association Computing Machinery, New York, 2010.

[16] B.J. Fogg, Persuasive technology: Using computers to change what we think and do, Morgan Kaufmann Publishers, San Francisco, 2002, pp. 1-5.

[17] R. Looije, M.A. Neerincx and F. Cnossen, Persuasive robotic assistant for health self-management of older adults: Design and evaluation of social behaviors, International Journal of Human-Computer Studies 68 (2010), 386-397.

[18] O. Blanson Henkemans, P.J. v.d. Boog, J. Lindenberg, et al., An online lifestyle diary with a persuasive computer assistant providing feedback on self-management, Technology and Health Care 17 (2009), 253-267.

[19] B. Fogg, A behavior model for persuasive design, Proceedings of the 4th International Conference on Persuasive Technology, ACM, 2009.

[20] A. Pantelopoulos and N.G. Bourbakis, A survey on wearable sensor-based systems for health monitoring and prognosis, IEEE Transactions on Systems Man and Cybernetics Part C-Applications and Reviews 40 (2010), 1-12. 